ANL-6881

Instruments

(TID-4500, 3 lst Ed.)

AEC Research and

Development Report

ARGONNE NATIONAL LABORATORY

9700 South Cass Avenue

Argonne, Illinois 60440

AN INTRODUCTION TO

MULTICHANNEL ANALYZERS

by

George S. Stanford

Reactor Physics Division

April 1964

Operated by The University of Chicago under

Contract W-31-109-eng-38

with the

U. S. Atomic Energy Commission 


\section{DISCLAIMER}

This report was prepared as an account of work sponsored by an agency of the United States Government. Neither the United States Government nor any agency Thereof, nor any of their employees, makes any warranty, express or implied, or assumes any legal liability or responsibility for the accuracy, completeness, or usefulness of any information, apparatus, product, or process disclosed, or represents that its use would not infringe privately owned rights. Reference herein to any specific commercial product, process, or service by trade name, trademark, manufacturer, or otherwise does not necessarily constitute or imply its endorsement, recommendation, or favoring by the United States Government or any agency thereof. The views and opinions of authors expressed herein do not necessarily state or reflect those of the United States Government or any agency thereof. 


\section{DISCLAIMER}

Portions of this document may be illegible in electronic image products. Images are produced from the best available original document. 
TABLE OF CONTENTS

Page

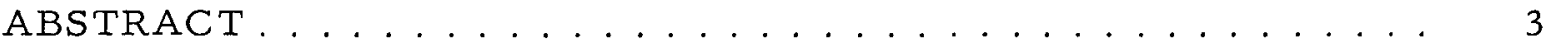

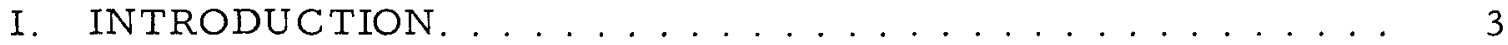

II. GENERAL PRINCIPLES OF OPERATION . . . . . . . . . . . 4

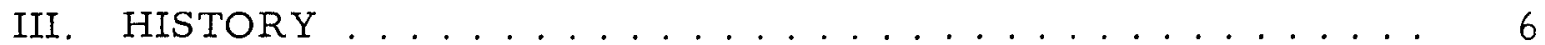

IV. FEATURES OF ONE-PARAMETER ANALYZERS. . . . . . . 9

V. PERFORMANCE OF SINGLE-PARAMETER

MULTICHANNEL ANALYZERS. . . . . . . . . . . . . . . 12

VI. MULTIPARAMETER ANALYZERS . . . . . . . . . . 13

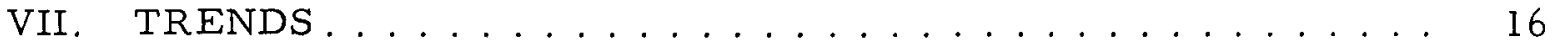

ACKNOWLEDGMENT . . . . . . . . . . . . . . . . 17

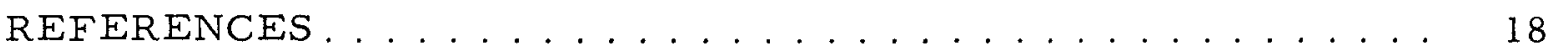




\section{LIST OF FIGURES}

No. Title Page

1. One-parameter Multichannel Analyzer............ 5

2. Analog-to-digital Conversion .............. 5

3. (a) Integral Bias Curve; and (b) Differential Bias Curve Obtained by Point-by-point Differentiation of the Integral Bias Curve...................... 6

4. Mechanical Kicksorter ................... 7

5. Coincidence-controlled Selective Storage ............ 11

6. Typical Experimental Setup with a Two-parameter Analyzer. . . . . . . . . . . . . . . . . . . . 14 


\title{
AN INTRODUCTION TO \\ MULTICHANNEL ANALYZERS
}

by

George S. Stanford

\begin{abstract}
A survey of the state of the art of multichannel pulse analysis is presented, intended chiefly for the reader with little prior knowledge of the field. There is no attempt to treat any topic in depth. The general principles of analysis of pulse height and time of flight are outlined, with consideration given both to single-parameter and multiparameter instruments. Memory systems, analog-to-digital conversion, and readout devices are discussed. Some of the features and limitations of modern analyzers are noted, and typical performance specifications are given. Although the main emphasis is on instrumentation, mention is made of some of the applications of single-and multiparameter analysis.
\end{abstract}

\section{INTRODUCTION}

In the last twenty years or so, multichannel pulse analysis has developed from the gleam-in-the-eye stage to its current state of healthy adolescence, and it is still growing rapidly. In this review the general principles will be mentioned, the story of its development will be outlined, and the current status will be surveyed. The treatment throughout will be superficial. For a more detailed discussion of almost any technical as pect of this subject, the reader is referred to the excellent monograph by R. L. Chase.(1)

First of all, what is multichannel analysis, and why is it used? The result of a multichannel analysis is a spectrum, in the form of a histogram (usually), with number of events given as a function of one or more of such parameters as the height of the pulse, or its shape, or its time of occurrence, or of the height, shape, or time of occurrence of coincident or otherwise time-related events. The application possibilities are legion. The chief use so far has been in nuclear physics, a typical application being the determination of the energy spectrum of particles from a radioactive source. This capability is increasingly being exploited in qualitative and quantitative chemical analysis of radioactive samples. A very sensitive detection of trace components can sometimes be made by neutron activation followed by 
pulse-height analysis. Even without chemical separation, such a method has been used to measure the manganese content of blood plasma, (2) where the total amount of manganese present was about $0.06 \mu \mathrm{g}$. There are further applications in such diverse fields as astrophysics (meteorite analysis), archeology, and criminology.

Another major use of multichannel analysis is in neutron time-offlight spectroscopy. A pulsed beam of neutrons, for example, can be directed through a sample to a neutron detector some distance away. By recording the time of detection of neutrons with respect to the time when the neutrons passed a reference position, the time-of-flight spectrum (and hence the energy spectrum) is recorded. The difference between the sample-in and sample-out spectra gives the energy spectrum of the neutrons absorbed or scattered by the sample. This information (the "total cross section") for different materials is important in nuclear reactor technology.

So far we have been discussing single-parameter analysis.* However, multiparameter analysis has for some time been used at the larger laboratories, and within the last year or so commercial multiparameter analyzers have started coming onto the market. There are many uses for multiple-parameter analysis. In determining a complex scheme of nuclear decay, one can examine the source with two detectors whose outputs serve as the two inputs to a two-parameter analyzer. When the events involving coincident pulses in the two counters are stored, there results a threedimensional record which is hard to display quantitatively, but from which can be extracted a great deal of information regarding the intercorrelation of the events in the two detectors. In other applications, one could study time-dependent phenomena by using as one parameter the time after some reference occurrence, such as an accelerator burst, with the pulse height of the detected event being the other parameter. Three-parameter experiments are also being performed although times required for a single experiment often are measured in days, and sometimes weeks. For more than three parameters, the rate of data acquisition tends to be so slow that most such experiments are impractical.

\section{GENERAL PRINCIPLES OF OPERATION}

The chief components of a one-parameter multichannel analysis system are shown in Fig. 1. Many variations on this theme have been used, but most modern one-parameter analyzers make use of the historic

* The terminology here is currently in a state of mild confusion, because the word "dimension" is sometimes used instead of "parameter." Some writers regard a two-parameter measurement as a three-dimensional one, the number of events being the third dimension, and some refer to it as being two-dimensional. The present writer feels that the result of such an experiment is unques tionably three-dimensional, but will for the most part avoid the issue by sticking to the unambiguous term "parameter." 
breakthroughs of Wilkinson(3) and of Hutchinson and Scarrott. (4) In the Wilkinson type of analog- (pulse-height-) to-digital converter (ADC), a small capacitor is permitted to charge up to the peak voltage of the pulse and is then discharged at constant current. While the discharge is in progress, "clock" pulses from a stable oscillator are counted by a scaler, the number of clock pulses counted being proportional to the time the capacitor took to discharge, and hence to the original height of the pulse (see Fig. 2). (Alternatively, a capacitor is sometimes charged linearly, until it reaches the height of the stretched input pulse. The result is the same.) By starting the ramp in synchronism with a clock pulse, a one-channel uncertainty in the start time can be avoided.

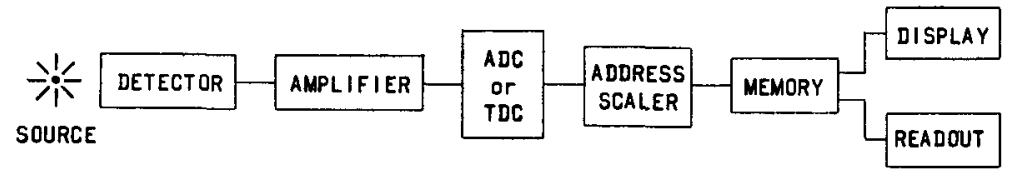

Fig. 1. One-parameter Multichannel Analyzer. $A D C$ and TDC stand for analog-to-digital converter and time-to-digital converter, respectively.

The number of clock pulses that occurred before the oscillator gate closed (this number is called the "address") is the number of the channel

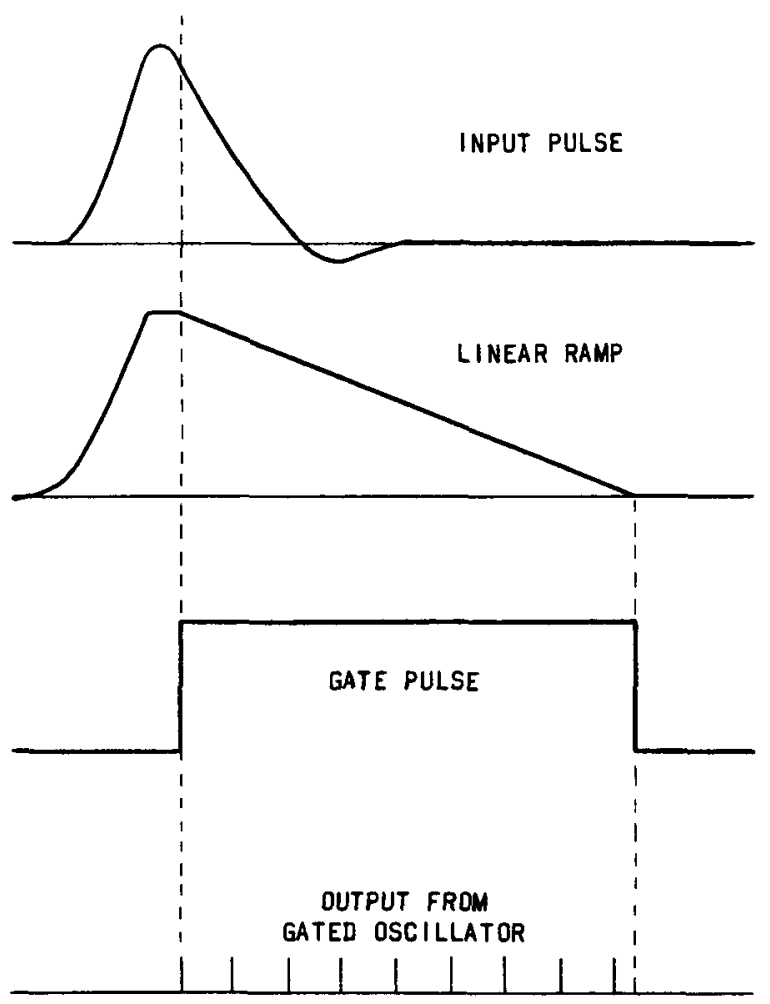

Fig. 2. Analog-to-digital Conversion that the count is to be stored in, and this is where Hutchinson and Scarrott contributed. In Wilkinson's system, the telephone-type registers used were not fast: he could store only about ten counts per second. Hutchinson and Scarrott introduced a computer-type memory for storing the pulse. Computer technology being rather primitive twelve years ago, Hutchinson and Scarrott in fact made do with an ultrasonic delay line (mercury), through which the memory contents circulated once every $1.2 \mathrm{~ms}$ in binary coding. Modern oneparameter analyzers tend to use memories consisting of threedimensional arrays of small ferromagnetic cores. Typically, a count is stored by reading out the contents of the appropriate channel, adding one, and re-storing the result. 
a much greater contribution to pulse-height-analyzer dead time is due to the conversion process. The clock oscillator circuits in general use give pulses at a 4 or $5 \mathrm{Mc}$ rate, making the conversion time for a pulse in channel 400, for instance, equal to 80 or $100 \mu \mathrm{s}$. It is, of course, necessary to prevent new pulses from entering the converter while it is converting, so that storage rates are limited to less than $10^{4}$ or so per second.

In time-of-flight work, the type of time-to-digital converter (TDC) used depends to some extent on the magnitude of the flight times involved.

For flight times running, say, $20 \mu \mathrm{s}$

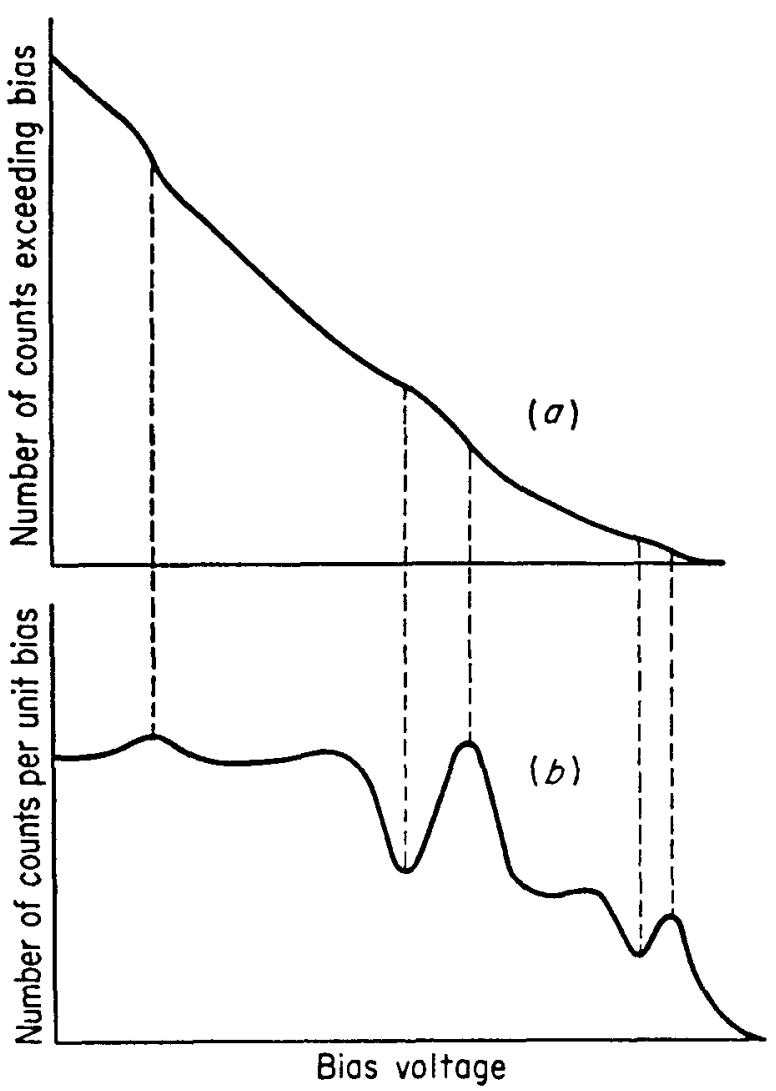

Fig. 3. (a) Integral Bias Curve; and (b) Differential Bias Curve Obtained by Point-by-point Differentiation of the Integral Bias Curve. (From Nuclear Pulse Spectrometry, by R. L. Chase, Copyright 1961. McGraw-Hill Book Company, Inc., used by permission.) or longer, it is feasible to start a clock oscillator at time zero (when the neutron starts its flight) and stop it when the neutron is detected. The count is then stored as before. For shorter flight times, ranging down into the nanosecond region, digital circuits are not fast enough. However, one can start a linear ramp at time zero, and stop it when the neutron arrives. The result is a pulse of amplitude proportional to the time of flight, and it can be analyzed by conventional methods of pulse-height analysis.

\section{HISTORY}

A pulse-height spectrum is a differential bias curve. Such a curve can in principle be derived from an integral bias curve (see Fig. 3), but since the process of numerical differentiation involves finding small differences between large numbers, the result has low statistical precision.

Multichannel analyzers were preceded by single-channel analyzers,

first described in 1940.(5) A single-channel analyzer is an instrument which counts only those pulses whose height lies within a voltage "window" (the "channel") defined by a pair of discriminators. A differential spectrum can be produced point by point by scanning the window, either continuously or stepwise, over the desired range. Unless the source is very active, it obviously takes about a hundred times as long to get a hundred-channel spectrum with a single-channel analyzer as with a 100 -channel analyzer. 
Thus, single-channel analyzers are not much used for that purpose any more, although they are very useful for experiments where it is desired to count pulses occurring in a pre-selected pulse-height interval.

Multichannel analyzers were probably first built in the early forties. The original open-literature report was published in 1947,(6) although some instruments apparently were described in earlier British Atomic Energy reports. (3) Naturally enough, the early multichannel analyzers were extensions of the single-channel-analyzer principle, $n+1$ discriminators being used to define $n$ pulse-height regions, or channels. Each channel of such a "multidiscriminator converter" can have its own scaler, and the discriminators are connected by anticoincidence circuits in such a way that a count is transmitted from a given discriminator only if the discriminator above it is not triggered. Because there is no analog-to-digital conversion, such instruments are still used for applications where very small dead time is important, but they tend to be bugged by problems of alignment and stability, and they also become large and cumbersome if many channels are incorporated. Twenty or thirty channels is typical, "while the largest in current use has 120 channels."(1) That channel-width adjustment can be tricky may be seen by considering that, if $1 \%$ precision in channel width is desired in a channel whose width is $1 \%$ of its position, then the upper and lower discriminators must be held to one part in $10^{4}$. This particular problem, however, can be alleviated by means of a pulse-increment technique,(1) which uses the same discriminator to define both the upper and lower limits of its channel: the input pulse is stretched, and then an increment equal to the desired channel width is added to it, producing a pulse with a step on top. The channel for the count to be stored in is determined by which discriminator is triggered by the addition of the increment.

For a while the name kicksorter was rather popular as a conveniently short synonym for "multichannel pulse-height analyzer." It is still used sometimes, but it never really

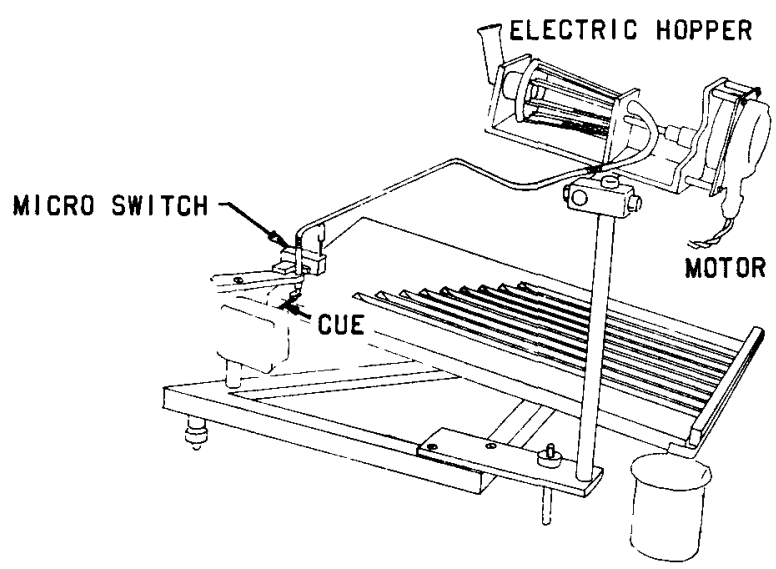

Fig. 4. Mechanical Kicksorter (From Reference 7) caught on. It was particularly appropriate, however, as a designation for a device built in 1949 by Frank, Frisch, and Scarrott (7) (see Fig. 4). It had thirty channels consisting of parallel slots milled in a sheet of polystyrene. A ball bearing rested on a pad in front of an electromechanical cue. When a pulse was detected, the cue would impart to the ball bearing an impulse proportional to the height of the pulse, and the ball bearing would come to rest at the lower end of the appropriate channel. A new ball bearing would 
automatically drop onto the launching pad to await the next event. The capacity was 100 counts per channel, and the instrument was reported to perform reliably at rates up to $6 \mathrm{cps}$.

Numerous other pulse-height-analyzing schemes have been used, some simple and some quite complex. One of the simple ones is the graywedge technique, (1) for which all that is required beyond the amplifier is a pulse stretcher, oscilloscope, camera, and gray wedge. The gray wedge, with its optical absorbance increasing linearly from zero at the left to some appropriate value at the right, fits over the oscilloscope screen. The pulse to be analyzed is stretched to produce a horizontal line across the oscilloscope screen, the height of the line being proportional to the height of the pulse. When a time exposure on high-contrast film is made of the oscilloscope screen, the film will contain the pulse-height spectrum (on its side), since the distance that the exposed portion of the film extends into the gray wedge, for any pulse height, depends upon how many sweeps occurred at that pulse height. The resulting intensity scale is logarithmic.

See Reference 8 for a convenient tabular summary of kicksorting schemes. See Reference 1 for more complete discussions of the schemes.

The remainder of this report is confined chiefly to the main line of development of the pulse-analyzing art. Following are some of the milestones:

(a) The principle of analog-to-digital conversion was introduced by Wilkinson(3) in 1950, as described in Section II. Although it has the disadvantage of a fairly long conversion time, the method is widely used because of the exceptional stability achievable in electronic oscillators. Since it is also possible to make highly linear voltage ramps, nowadays problems of stability and linearity tend to show up first in the detectors and amplifiers. For many applications where high storage rates are necessary, the problem of the long conversion time can be alleviated by various buffer-storage schemes, to be mentioned further in Section VII, or by using a multidis criminator converter.

(b) The addition of the computer-type memory was made by Hutchinson and Scarrott $(4)$ in 1951 , and was mentioned in Section II. Immediately Wilkinson's storage rate of ten per second was increased to 1600 per second. When the magnetic-core memory replaced the acoustic delay line, storage times of 10 to $30 \mu \mathrm{sec}$ became possible, permitting (neglecting the much longer conversion time) storage rates of up to $10^{5} / \mathrm{sec}$.

(c) The advent of transistors was very important. The practical number of channels for vacuum-tube circuitry was limited by sheer physical size and component reliability. With vacuum tubes a 100-channel analyzer occupied more than a 6-ft rack, whereas one can now buy a 400-channel analyzer with cathode-ray tube (CRT) display that can be carried in one 
hand.* (Readout equipment still takes up a lot of room.) It also frequently happened that a many-channel, vacuum-tube analyzer was undergoing repair a large proportion of the time, whereas the transistorized circuitry has proven to be much more reliable.

(d) Multiparameter analyzers have added new possibilities. The advent of multichannel, one-parameter analyzers, by speeding up data acquisition by one to three orders of magnitude, made possible many experiments which would have been virtually impossible with single-channel analysis. A further jump of the same general magnitude has occurred with the utilization of multiparameter analyzers, which will be discussed in a later section.

\section{FEATURES OF ONE-PARAMETER ANALYZERS}

The eight or more manufacturers** selling pulse-height analyzers in the United States and Canada offer a variety of instruments. In oneparameter analyzers the number of channels ranges from 100 to 800 , with capacities of $10^{5}$ or $10^{6}$ counts per channel. Prices range from about $\$ 5000$ for small units with simple readout equipment, to well over $\$ 20,000$ for large systems including a variety of operational and readout options. In this section, some of the currently available features and options will be mentioned.

Most instruments offer selective storage - the ability to divide the memory into halves, quarters, or eighths, and to "route" the input to any desired segment. Thus it is possible to store up to eight spectra in the same memory.

Background subtraction can be accomplished by first storing background in the negative mode, and then counting the same for the same length of time in the positive mode. However, the feature of memory transfer, combined with selective storage, permits one to subtract the same background count over and over, by storing it in one segment of the memory, in

* Technical Measurements Corporation, 441 Washington Avenue, North Haven, Connecticut.

* * The chief (perhaps only) American manufacturers of multichannel analyzers are:

(1) Linear Alpha, Inc., 823 Emerson Street, Evanston, Illinois 60201.

(2) Nuclear Data, Inc., 3833 West Beltline Highway, Madison 5, Wisconsin.

(3) Packard Instrument Company, Inc., Box 428, LaGrange, Illinois.

(4) Radiation Counter Laboratories, Inc., 5121 West Grove Street, Skokie, Illinois.

(5) Radiation Instrument Development Laboratory, Inc., 4501 West North Avenue, Melrose Park, Illinois.

(6) Technical Measurements Corporation, 441 Washington Avenue, North Haven, Connecticut.

(7) The Victoreen Instrument Company, 5806 Hough Avenue, Cleveland 3, Ohio.

(8) Tech-Systems, Inc., 33 Danbury Road, Wilton, Connecticut. 
the negative mode, and then transferring it nondestructively (i.e., without erasing the original record) to the segment in which one will store the spectrum of the sample.

With automatic programming, it is possible to cause the instrument to perform repetitively a sequence such as this:

(1) Transfer, nondestructively, into the first half of the memory the negative-mode background that has been stored in the second half.

(2) Count the sample for preset "live time" (see below).

(3) Read out the resulting spectrum, destructively, into whatever readout device is provided.

(4) Repeat.

Thus one can automatically obtain successive spectra of the same sample, or, in conjunction with an automatic sample changer, spectra of a series of samples.

Spectrum stripping is the process of analyzing a spectrum by subtracting from it, one by one, the spectra of known components. Some sys tems permit this to be done as follows. First, the standard spectra of the components to be looked for must be individually stored on paper tape. Then the spectrum to be analyzed is stored in one-half of the memory. The standard spectrum of component $A$ is then read from the tape into the other half of the memory. A computer in the analyzer permits any desired multiple of the standard spectrum to be subtracted from the unknown. The operator observes the process on the oscilloscope display, and subtracts in steps until the features of component $A$ seem to have been cancelled. The process can then be repeated for the next component. An important point to be aware of here is that, although ordinate (intensity) multiplication of the standard spectrum is provided, abscissa (pulse-height) multiplication is not. It is therefore imperative that the standard and unknown spectra have the same resolution, with the same peaks occurring at the same locations. A difference of even one-half per cent in peak position can cause unsatisfactory results. Computer programs for taking care of the problem of pulse-height adjustment have been reported. $(9,10)$

Most of these analyzers have two or more inputs, with internal routing, making it possible to store several spectra simultaneously (but not simultaneous pulses, of which at least one must usually be rejected), with each input routed to its own memory segment. (In practice, it appears that some manufacturers find it hard completely to eliminate cross-talk storage of the occasional pulse in the wrong memory segment.) Sometimes it is possible to apply an external "routing signal" to cause the analyzer to store the pulse in a particular memory segment. An application of such a system is shown in Fig. 5. (11) 


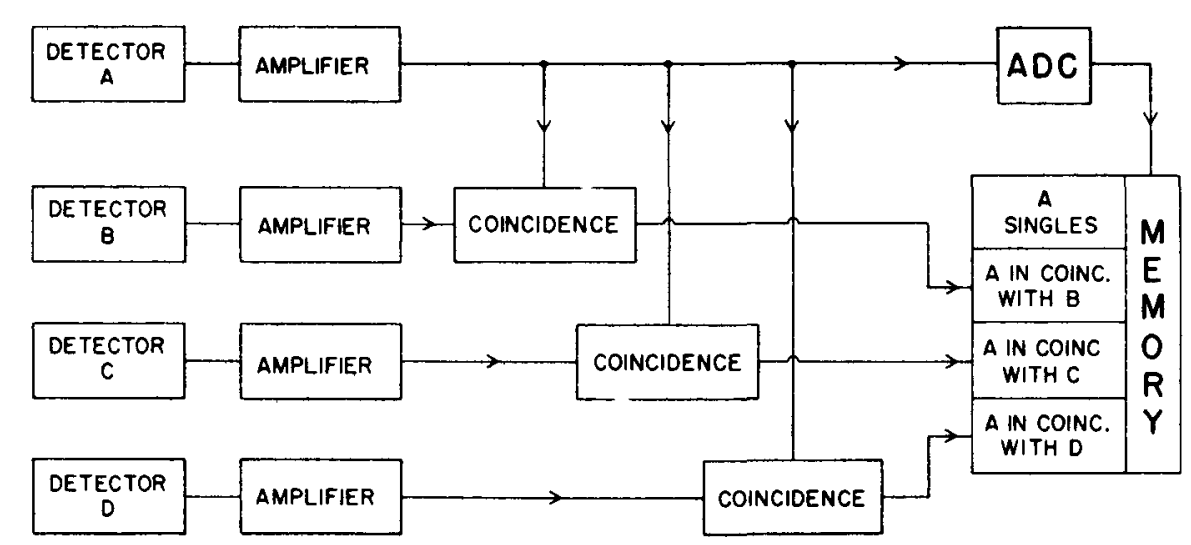

Fig. 5. Coincidence-controlled Selective Storage. Signals from Detector A are directed to different memory sections depending on which, if any, of the other detectors produced time-coincident signals. (From Reference 11)

All of the analyzers can display the memory contents on a cathoderay tube (CRT) while no input is being analyzed. In addition, most of them have a live display, which shows each pulse as a brief flash on the CRT screen as it is being stored. If the counting rate is fairly high, one can watch the spectrum growing.

Sometimes a switch is provided to render the ordinate (relative intensity scale) either linear or logarithmic. The latter has the advantage that the displayed spectral shape is independent of the counting time. This is particularly useful in visually comparing a spectrum stored in one segment of the memory with a similar one in another segment, utilizing a feature which permits the simultaneous, superimposed display of more than one segment.

While the analyzer is busy converting and storing a pulse, it is rendered insensitive to new input pulses. For accurate quantitative work it is often insufficient to know the "clock-time" duration of the count: one needs to know the live time, which is the clock time minus the dead time the actual length of time the analyzer was ready to accept input pulses. This can be measured rather simply by means of an accurate, usually crystal-controlled, continuously running oscillator, whose pulses are counted only if the input is "live" when the pulse occurs. Thus, within statistics, the number of live-timer pulses accepted is proportional to the live time, provided that the pulses to be analyzed arrive at random times. If the pulses arrive in bursts, as for instance from a pulsed accelerator, the live timer will give a misleading indication.

In most analyzers, a meter continuously indicating per cent dead time either is provided or can easily be added. Some instruments print out the live time of the run as the contents of the first data channel, and some do not. 
The option of multichannel scaling is offered by most of the instruments. With this feature in operation, the channels become time channels instead of pulse-height channels. At the start of counting, all input pulses above the lower discriminator setting are registered as counts in channel 1. Channel-advance signals, internally or externally generated, switch the counting to channel 2, and so on, at a predetermined rate. The result is a record of the counting rate as a function of time such as one would want for determining the half-life of a radioactive source.

For quantitative analysis, the area under a peak is often a more reliable indication of its intensity than the amplitude at the maximum. Some instruments can be programmed to integrate under peaks, summing the counts between selected pulse-height limits.

All types of readout equipment are available: oscilloscope camera, punched paper tape, typewriter, parallel paper-tape printer, magnetic tape, $x-y$ plotter. The punched-tape and magnetic-tape readouts can be made compatible with the input required by computers.

If a prospective user intends to extract quantitative amplitude information from analog output, such as $\mathrm{x}-\mathrm{y}$ plotter or CRT display, he should be aware that some analyzers "decode" for display only the two most significant digits, while the other decode three. With two-digit decoding, there is the possibility of a maximum error of $10 \%$ in the ordinate for any one channel, since 109 counts appear as 100 counts, 1099 as 1000 , and so on.

\section{PERFORMANCE OF SINGLE-PARAMETER MULTICHANNEL ANALYZERS}

In this section some quantitative indication will be given of the performance of modern, commercial, one-parameter multichannel pulse-height analyzers. The numbers given are indicative only, since there are differences between instruments. In most cases they represent specifications set by the manufacturer.

Integral Linearity: $\pm 0.5 \%$ of full scale, over top $97 \%$ of scale. Integral linearity is the maximum deviation of a channel position from where it should be, as a percentage of full scale.

Differential Linearity: $\pm 2 \%$, over top $97 \%$ of scale. Differential linearity is the maximum deviation of channel width from the average for all channels. Channel-width variations can cause spurious structure to appear in the spectrum.

Dead Time: $(30+5.0 \mathrm{~N})$ microseconds, where $\mathrm{N}$ is the number of the channel that the count is to be stored in. From this, one can tell that 
in this particular case the ADC clock pulses come at a $2-\mathrm{Mc}$ rate, and that $30 \mu \mathrm{sec}$ are required to store the count and allow the circuitry to settle down in preparation for the next pulse.

Maximum Input Rate: Up to 50,000 counts/sec, "with negligible spectrum distortion." Nevertheless, for quantitative work with present-day circuitry, the input rate should usually be kept well below $10^{4}$ counts $/ \mathrm{sec}$.

Input Pulse-shape Requirements: Variable from one instrument to another, but in general it can be said that pulses of the same height but with very different rise or fall times will tend not to count in the same channel. Many instruments do not function in top form if the pulse rise time is longer than a microsecond.

Live Timer Accuracy: Better than 1/2\%, up to 5000 counts/sec.

Multiple-input Cross-talk Rejection: Some instruments are better than others (see Section IV above). A prospective purchaser to whom this is important should himself test the instrument before purchase. (In fact, this should probably be said of any of the specifications.)

Power Consumption: Less than $50 \mathrm{~W}$.

\section{MULTIPARAMETER ANALYZERS}

As remarked before, the advent of multiparameter analyzers has greatly speeded data-taking in certain types of experiments, and has, in fact, made possible a new range of experiments that could probably not be done otherwise. For the most part, multiparameter analyzers use the principles of operation developed for single-parameter instruments, the chief difference being in the required memory size. The magnitude of the difference can be appreciated by noting that for 100 -channel coverage in each of $\mathrm{n}$ dimensions, one needs $100^{\mathrm{n}}$ channels, making $10^{4}$ channels for two dimensions and $10^{6}$ for three - and frequently 100 -channel resolution is not good enough. analysis. (11)

Figure 6 shows an experimental arrangement for two-parameter

Memories for multichannel analyzers can be of two types, accumulating and open-ended. As examples of the former we have the commonly used magnetic-core memory and the less widely used rotating magnetic drum. (12) In such a memory, each channel has its own set of locations, and whenever a count occurs, the contents of the appropriate channel are increased by one. A typical open-ended system records all the information 
about each event digitally on magnetic tape (or occasionally on punched paper tape, moving film, etc.) An accumulating memory has the distinct advantage that its contents can be interrogated at will, to see how the experiment is going, whereas the open-ended tape must be processed later in auxiliary equipment.

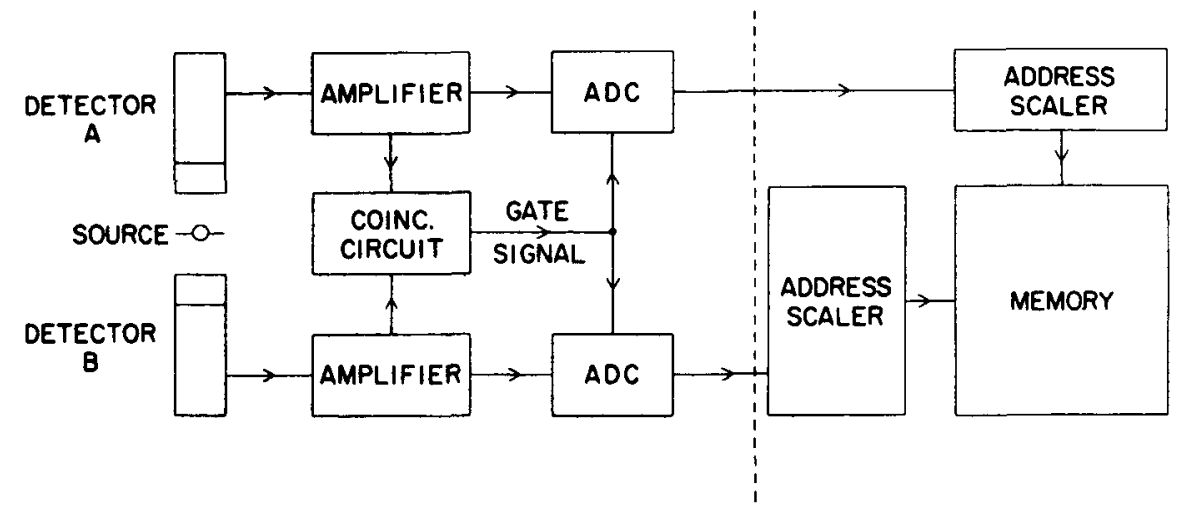

Fig. 6. Typical Experimental Setup with a Twoparameter Analyzer (From Reference 11)

For memories of large capacity, however, open-ended systems are much more economical. The cost per bit for magnetic-core memories has been given $(13)$ as 10 to 30 cents. Consider now that to be able to accumulate $10^{5}$ counts in a channel (in binary coding), the channel must have a 17 -bit capacity (that is, 17 cores per channel). Using the examples given above, $10^{4}$ such channels would cost from $\$ 17,000$ to $\$ 51,000$, and $10^{6}$ channels $\left(2 \times 10^{7}\right.$ bits) would be completely out of reach. Magnetic drums are somewhat cheaper, costing only "a few cents per bit,"(13) but for large memories there seems at the moment to be no practical alternative to magnetic tape. At 200 bits per inch, a $3600-\mathrm{ft}, 25$-level tape could hold about $2 \times 10^{8}$ bits. By contrast, what is probably the largest commercially offered core memory for pulse analyzers has about $4 \times 10^{5}$ cores.*

A commercial instrument is being developed which combines the large capacity of magnetic-tape storage with some ability to monitor the progress of the experiment.** It is done by storing simultaneously in a 4000-channel core memory a preselected portion of what is being stored on the magnetic tape. The core memory can provide either a low-resolution view of the entire experiment, or a high-resolution view of only a portion of the data.

Many descriptions of multiparameter experimental setups have appeared in the literature. $(12,14-19)$ (The references given are representative, not comprehensive.) As an example of one of the more elaborate

*The Victoreen Instrument Company, 5806 Hough Avenue, Cleveland, Ohio.

**Nuclear Data, Inc., 3833 West Beltline Highway, Madison 5, Wisconsin. 
arrangements, the Argonne National Laboratory three-parameter analyzer $(17,19)$ will be briefly described. This instrument achieves over 33 million $\left(2^{25}\right)$ channels by storing each event on a 25 -track magnetic tape, $1 \frac{1}{4}$ in. wide. For one of the parameters 512 channels are available, leaving 256 for each of the other two. After the storage of each event, the tape is advanced $0.01 \mathrm{in}$. to be ready for the next one. A one-event buffer storage gives a two-event resolving time of $230 \mu \mathrm{sec}$, determined largely by the time required for analog-to-digital conversion. At high counting rates, the tape moves continuously. Maximum storage rate is $170 / \mathrm{sec}$, which, because of the usual slow rate of detection of three-parameter events, is satisfactory.

The system was designed explicitly for use in studies of the gammaray spectra that result from the capture of neutrons in resonances.(18) A neutron beam from a reactor is divided into bursts by a rapidly rotating "chopper." Twenty-five meters from the chopper, a sheet of the material under investigation intercepts the beam, and when a neutron is absorbed, the resulting excited nucleus usually gets rid of its excess energy immediately by emitting several gamma rays. Two large sodium iodide scintillation crystals detect some of these gamma rays. When a coincidence occurs, its time of occurrence is recorded on the tape (using the time-todigital conversion process previously described) as one of the three parameters, the other two being the pulse heights in the two detectors. The time of occurrence gives the energy of the neutron which caused the event, and this can be correlated with information about the nuclear structure as revealed by the two pulse heights.

After the data have been accumulated (typically in one or two days of running time), the tape is transferred to a "search station," which might be described as a special-purpose digital computer. The search station permits four two-dimensional (i.e., one-parameter) spectra to be read simultaneously from the tape into a 1096-channel core memory in about $3 \frac{1}{2} \mathrm{~min}$, and the memory contents can be displayed on an oscilloscope or read out for a permanent record. Each such spectrum is the result of imposing operator-selected conditions on two of the three parameters - say a particular neutron time of flight, and a selected pulse-height interval in one of the detectors - with the recording of the entire spectrum in the third parameter, of events which met the two imposed criteria. Since reading out all of the information contained on the tape would lead to a hopeless mountain of data, the experimenter must do his best to select only the pertinent information.

For more details on the construction and performance of this installation, the reader is referred to References 17 and 18. In Reference 20 will be found a fuller discussion of some types of information being obtained with three-parameter analysis. 


\section{TRENDS}

With the advance of general technology, there are continuing demands for improvement of various aspects of the performance of multichannel analyzers. At the present moment, there is probably somebody ready to exploit a significant improvement in any characteristic that one could name: storage speed, readout speed, conversion time, physical size, stability, memory capacity, cost.

Perhaps the currently most pressing need is for reduction of analogto-digital conversion time, a need which is brought about primarily by the large pulsed accelerators. Frequently an experiment requires that many events per accelerator pulse be accepted for analysis, and in the present state of the art not very many events per accelerator pulse can be directly converted by a Wilkinson-type ADC. Several schemes for temporary ana$\log$ storage have been developed; $(21-26)$ they use the time between accelerator bursts for the leisurely conversion of the stored analog information. Some of these methods have been quite successful for their intended purpose. However, the resolution obtainable with any form of analog storage is inherently limited, and this limitation can be especially troublesome when high-resolution solid-state detectors are used.

In fact, the introduction of these detectors, with resolution approaching $0.5 \%$, has already tested the stability of existing circuitry, stimulating the invention of feedback methods for drift cancellation. $(27,28)$

For some experiments, the time required to store a count in the memory is a limiting factor. In the case of a core memory, this time in commercial instruments is 20 to $30 \mu \mathrm{sec}$, which includes settling-down time after storage has been accomplished. Dual address registers or other mechanisms providing one-event buffering are often used, which alleviates the problem by permitting two closely spaced events to be recorded, but the counting rate for more than two pulses is still limited by the memory cycle time. Very recently, new instruments have come on the market with greatly reduced storage time. One* is a 40 -channel device with a storage time of only $1 \mu \mathrm{sec}$. It has a 800 -bit memory, and seems to be intended primarily for temporary storage of events occurring during an accelerator pulse. Another** is a 256-channel analyzer with a 100-Mc ADC and a stated average dead time of $3 \mu \mathrm{sec}$; its memory is of the newly developed "thin film" variety, ${ }^{(29)}$ whose total storage time is said to be $0.6 \mu$ sec.

*The "Syntec/150," a product of Linear Alpha, Inc., 823 Emerson Street, Evanston, Illinois 60201.

**Radiation Instrument Development Laboratory, Inc., 4501 West North Avenue, Melrose Park, Illinois. 
There seems to be hope of a further reduction in storage time by an order of magnitude or so, perhaps by cryogenic techniques. (13)

The development of digital readout equipment has tended to lag behind other aspects of multichannel analyzer development, in freedom from breakdown $(18,30)$ as well as in speed. An automatic typewriter prints about one channel per second, a parallel printer about five channels per second. For subsequent computer use, however, magnetic-tape readout can be accomplished very rapidly, rates of the order of 1000 channels per second being reasonable. Analog readout can be accomplished by photographing an oscilloscope pattern or by means of an $x$-y plotter. The latter operates at a speed of about 1.5 channels per second. An interesting new type of digital readout device has recently been offered for sale with the trade name "Optikon."* It is stated that it provides a readout speed of about 20 channels per second, the output being a Polaroid picture with the contents of up to 1024 channels printed on it in ordinary Arabic numerals.

One of the chief problems with multiparameter analysis is the mountainous volume of data that tends to collect. This is leading to the increased use of computers for data analysis. There is, for example, a setup at Argonne National Laboratory (in the Applied Nuclear Physics Section of the Reactor Physics Division) designed to feed the address information from the analog-to-digital converters directly into a computer: for the measurement of neutron cross sections, the computer is programmed to produce the calculated cross section a matter of minutes or less after the data taking has ceased.(31)

It would be out of keeping with the times not to mention that multichannel analyzers are going out into space.(32) They are, of course, highly miniaturized, typically with only a small number of carefully selected channels. One, for use in Ranger moon probes, has 32 channels with a capacity of 56,535 counts per channel.(33)

Regarding possible future developments, it has been predicted that in the next few years there will be order-of-magnitude improvements in smallness of electronics, circuit operation speeds, and bit-packing density on magnetic tape. (34) In addition, particularly for multiparameter analysis, there exists the possibility of improving memory utilization by increased use of preselection which automatically rejects many of the large number of uninteresting events. (35) And an increasingly close tie-in between multichannel analysis and electronic computers can be expected.

\section{ACKNOWLEDGMENT}

The writer is indebted to Dr. Edgar Bennett for his constructive criticism of the manuscript.

*Nuclear Data, Inc., 3833 West Beltline Highway, Madison 5, Wisconsin. 


\section{REFERENCES}

1. R. L. Chase, Nuclear Pulse Spectrometry, McGraw-Hill Book Company, Inc., New York (1961).

2. D. C. Borg et al., Selective Radioactivation and Multiple Coincidence Spectrometry in the Determination of Trace Elements in Biological Materials, Intern. J. Appl. Radiation and Isotopes, 11, 10-29 (1961).

3. D. H. Wilkinson, A Stable Ninety-nine Channel Pulse Amplitude Analyzer for Slow Counting, Cambridge Phil. Soc., 46, 508-518 (1950).

4. G. W. Hutchinson and G. G. Scarrott, A High Precision Pulse-height Analyzer of Moderately High Speed, Phil. Mag., 42, 792-806 (1951).

5. Arthur Roberts, A Differential Pulse Amplitude Selector, Rev. Sci. Instr., 11, 44-45 (1940).

6. H. F. Freundlich, E. P. Hincks, and W. J. Ozeroff, A Pulse Analyzer for Nuclear Research, Rev. Sci. Instr., 18, 90-100 (1947).

7. S. G. F. Frank, O. R. Frisch, and G. G. Scarrott, A Mechanical Kicksorter (Pulse Size Analyzer), Phil. Mag., 42, 603-611 (1951).

8. R. V. Gasstrom, A Versatile Pulse Height Analyzer Utilizing PhotoPick-Up Sorting Off a CRT Screen, Nuclear Electronics, Part I. Proc. Intn'1. Symposium on Nuclear Electronics, Organized by the French Society of Radioelectricians, Paris, 1958, pp. 317-331.

9. R. L. Heath, Recent Developments in Scintillation Spectrometry, IRE Trans., NS-9, No. 3, 294-304 (1962).

10. H. I. West, Jr. and B. Johnston, Scintillation Counter Gamma Spectra Unfolding Code for the IBM-650 Computer, IRE Trans., NS-7, Nos. 2-3, 111-115(June 1960).

11. R. L. Chase, Recent Developments in Multichannel Pulse-height Analyzers, IRE Trans., NS-9, No. 3, 257-259 (June 1961)(BNL-5993).

12. R. L. Chase, A Two-dimensional Kicksorter with Magnetic Drum Storage, IRE National Convention Record, Part 9, pp. 196-201 (1959).

13. T. K. Alexander, F. S. Goulding, and W. D. Howell, General Survey on Multichannel Analyzer Storage Techniques for Nuclear Experiments, Proc. of Symposium on Neutron Time-of-Flight Methods, Brussels, July 24-27, 1961, pp. 449-455. European Atomic Energy Community (September 1961). 
14. M. Birk, T. Braid, and R. Detenbeck, Two-dimensional Pulse-height Analyzer, Rev. Sci. Instr., 29, 203-209 (1958).

15. T. K. Alexander and L. B. Robinson, Three-dimensional Coincidence Analyzer Uses 900 Addresses, Nucleonics 20(5), 70-74 (May 1962).

16. C. D. Goodman, G. D. O'Kelley, and D. A. Bromley, A 20,000-channel Pulse-height Analyzer with Two Co-ordinate Address, Proc. Symposium on Nuclear Instruments, Academic Press, Inc., New York, 1962, pp. $189-196$.

17. C. C. Rockwood and M. G. Strauss, Three-parameter Multichannel Recorder-Analyzer System, Rev. Sci. Instr., 32, 1211-1221 (1961).

18. L. M. Bollinger, The Argonne 3-parameter Analyzer, Proc. of Conf. on Utilization of Multiparameter Analyzers in Nuclear Physics, Grossinger, New York, November 12-15, 1962, USAEC NYO-10595, pp. 59-69 (1963).

19. M. G. Strauss, Two-parameter Multichannel Pulse-height Analyzer, Proc. of Conf. on Utilization of Multiparameter Analyzers in Nuclear Physics, Grossinger, New York, November 12-15, 1962, USAEC NYO-10595, pp. 95-98 (1963).

20. H. E. Jackson and L. M. Bollinger, Isotopic Identification of Neutron Resonances of $\mathrm{Cd}, \mathrm{Sb}, \mathrm{Os}$, and Pt from Capture Gamma-ray Spectra, Phys. Rev., 124, 1142-1152(1961).

21. L. Costrell and R. Brueckmann, Charge Storage Pulse-height Analyzers for Use with Pulsed Accelerators, Nuclear Instr., 3, 350-358 (1958).

22. J. Cunningham, A Multichannel Pulse Height Analyzer Using a CR Storage Tube, Nat. Bur. Standards Report No. 3258 (April 1954).

23. D. F. Hebbard, Consecutive Amplitude Analysis for Simultaneous Pulses, Nuclear Instr. and Methods, 16, 267-274 (July 1962).

24. K. Kandiah, High Speed Intermediate Storage Systems, Proc. Symposium on Nuclear Instruments, Harwell, September 1961, pp. 168-177, Academic Press, Inc., New York (1962).

25. G. F. Pieper, A Fast Storage System for Multichannel Pulse Height Analysis, Nuclear Electronics, Part I. Proc. Intn'l. Symposium on Nuclear Electronics, Organized by the French Society of Radioelectricians, Paris, 1958, pp. 225-231. 
26. F. Wells, The Use of Electrostatic Storage Tubes for Fast Nuclear Data Storage, Fast Pulse Techniques in Nuclear Counting, Proc. 2nd Symposium on Advances in Fast Pulse Techniques for Nuclear Counting, Lawrence Radiation Laboratory, Berkeley, California, February 1959, p. 29, UCRL-8706.

27. R. L. Chase, A Servo Stabilized Analogue-to-digital Converter for High Resolution Pulse-height Analysis, IRE Transactions, NS-9, No. 1, $119-122(1962)$.

28. J. A. Ladd and J. M. Kennedy, A Digital Spectrum Stabilizer for Pulse Analyzing Systems, CREL-1063(1961).

29. A. A. Fleischer and E. Johnson, New Digital Conversion Method Provides Nanosecond Resolution, Electronics, pp. 55-57(May 3, 1963) Reference is made to Burroughs Corp., Thin Film Memory, Bull. No. 1007 and Addenda (1961).

30. C. H. Westcott, General Survey on Multichannel Analyzer Techniques. Practicality of Alternatives Including Recording Techniques, Proc. of Symposium on Neutron Time-of-Flight Methods, Brussels, July 24-27, 1961 , pp. 523-528, European Atomic Energy Community (September 1961).

31. J. F. Whalen, J. W. Meadows, and R. N. Larsen, "On-Line" Operation of a Digital Computer in Nuclear Physics Experiments, Rev. Sci. Instr., 35, 682-90 (June 1964).

32. J. R. Wolff and R. M. Ravenesi, Multichannel Analyzers in Space, Nucleonics, 20(10), 58-60 (Oct 1962).

33. M. A. Van Dilla and E. C. Anderson, Lunar Composition by Scintillation Spectroscopy, IRE Trans., NS-9, No. 3, 405-412 (June 1962).

34. F. H. Wells, Comments at end of Reference 30.

35. C. C. Rockwood, General Survey on. Simultaneous Recording Techniques, Proc. of Symposium on Neutron Time-of-Flight Methods,

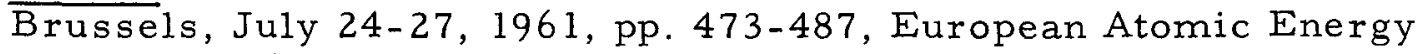
Community (September 1961). 